\title{
Event-Driven Energy-Harvesting Wireless Sensor Network for Structural Health Monitoring
}

\author{
Ming-Yuan Cheng, Yan-Bin Chen and Hung-Yu Wei \\ Department of Electrical Engineering \\ National Taiwan University, Taipei, Taiwan \\ Email: r99921033@ntu.edu.tw,d96921030@ee.ntu.edu.tw \\ and hywei@cc.ee.ntu.edu.tw
}

\author{
Winston K.G. Seah \\ School of Engineering and Computer Science \\ Victoria University of Wellington \\ New Zealand \\ Email: winston.seah@ecs.vuw.ac.nz
}

\begin{abstract}
Rare catastrophic events, like earthquakes, can cause substantial damage in a short span of time. Data on the level of stress sustained by buildings and other critical infrastructure acquired during the event can significantly help in post-disaster recovery and assessment of buildings' structural integrity. While installing sensors to acquire such data is not difficult, ensuring that there is power to drive the sensors at the critical moment of the event is a challenge. In this paper, we propose an event-driven energy-harvesting (EDEH) wireless sensor network (WSN) in which the sensors are powered by the energy harvested from the consequence of the event, e.g. buildings shaking during an earthquake. The scarce amount of energy harvested during the short event occurrence time poses great challenges for the medium access control (MAC) design, which is the focus of our research. Furthermore, when all sensors harvest energy from the event, they become active simultaneously leading to serious channel contention problems. As such, we first examine the amount of harvestable energy and then show analytically that our MAC protocol is able to provide higher packet delivery ratio than conventional wireless technology, e.g. IEEE802.15.4.
\end{abstract}

Index Terms-Event-Driven Energy-Harvesting; Structural Health Monitoring(SHM); Medium Access Control;

\section{INTRODUCTION}

In an earthquake, buildings and critical infrastructure are subjected to different levels of stress that can result in severe structural damages that render a building and its surrounding area unsafe. Such rare catastrophic events can cause substantial damage in a short span of time. Engineers are called upon to assess the damage and structural integrity of the building at short notice and often can only rely on visual inspection together with their years of professional experience to make critical decisions. These decisions often have major influence on the post-disaster recovery process.

Structural engineers can benefit from a simple sensing system, which monitors the level of vibrations that different parts of a building have been subjected to, to help them in their assessment of the potential damage suffered by the building. This can help them focus on the areas that have been sustained the highest level of vibrations and stress. Such sensors need to be operating at the time when the earthquake occurs. At other times, they need not operate at all. The issue of supplying power to the sensors at the right time arises. One approach is to connect the sensors to a constant source of power using wires. However, the wires may be damaged or severed during

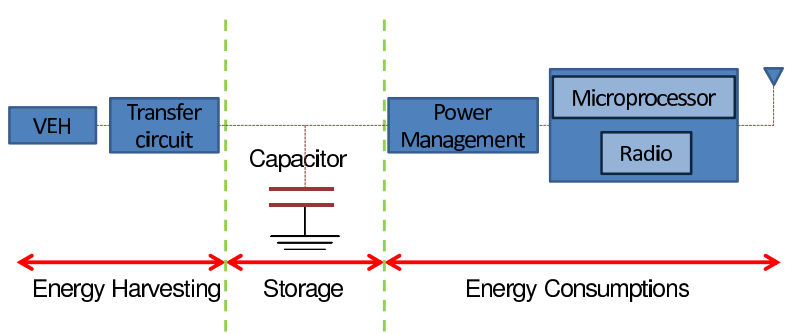

Fig. 1: Wireless sensor nodes functional architecture

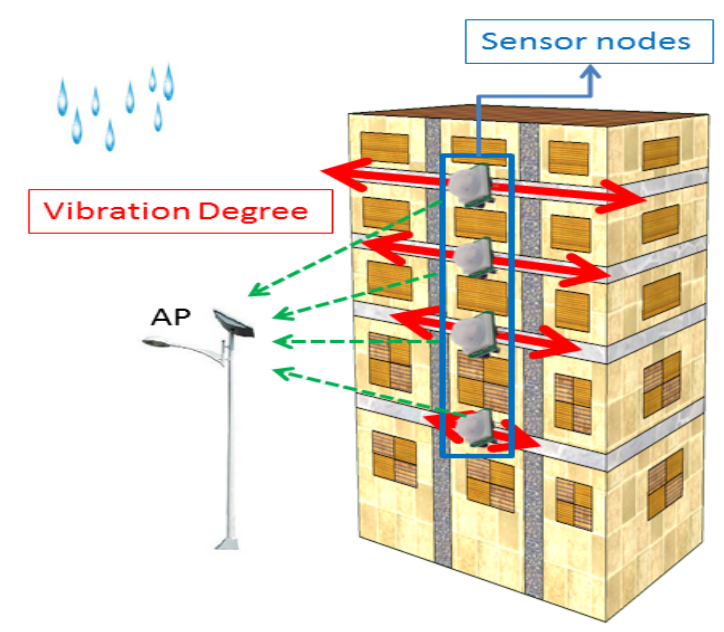

Fig. 2: EDEH WSN for SHM during earthquakes

the earthquake and leave the sensors without power at the most critical moments. Another approach is to use portable power sources like batteries. This then requires regular maintenance to replace the batteries which can be infeasible when these sensors are deployed in locations that are not easily accessible.

To address the unique energy resource requirements of such structural health monitoring (SHM) applications, we propose the event-driven energy-harvesting (EDEH) wireless sensor network (WSN) in which the sensors are powered by vibration energy harvesting (VEH) - the energy harvested from the consequence of the event, i.e. vibrations from buildings shaking during an earthquake. We use the phenomenon that we need to measure to power the sensor itself. The key functional 
blocks in the proposed sensing device is shown in Fig. 1. When buildings shake, the energy harvested from the vibrations will turn on the device to sense and transmit packets containing the sensor identifier and data from other sensing inputs, e.g. accelerometer, to a nearby collection/access point (AP), as shown in Fig. 2. The short event duration severely limits the amount of energy that can be harvested, and this poses great challenges for the medium access control (MAC) protocol. Another aggravating condition is that all sensors harvest energy from the event and become active almost simultaneously leading to serious channel contention problems. On the other hand, data from sensors located in close proximity to one another are highly-correlated. Thus, a key consideration in our design is to minimize the transmission of packets carrying highly-correlated data.

This paper is organized as follows. First, we briefly survey MAC protocols for WSNs powered by energy harvesting in Section II. Section III discusses empirical results of energy harvesting $(\mathrm{EH})$ rates combined with historical data of real earthquakes and power dissipation of the components in a typical EDEH device. Next, in Section IV, we describe our MAC protocol which uses the IEEE802.15.4 design as a basis with critical modifications needed to handle the unusual characteristics of SHM during earthquakes and the stringent energy resource constraints. Finally, we validate the analytical models using extensive simulations incorporating empirical data from Section III and compare the performance of our protocol against IEEE 802.15.4 in Section V. Section VI concludes the paper.

\section{RELATED WORK}

In this section, we briefly survey work done on WSNs using $\mathrm{EH}$ for power, focusing on MAC-related efforts. Firstly, challenges in MAC protocol design for wireless sensor networks using energy harvesting technologies have been discussed by Seah et al. [1], highlighting the issues arising from unpredictable energy availability. Kansal and Srivastava [2] provide two design approaches, namely, learning the environment by using prediction filter, then using the information to predict how much energy is harvestable, and sharing network-wide information to facilitate devices in making in-network decisions. Subsequently, Kansal et al. [3] provide a comprehensive description of energy harvesting and adaptive duty cycle models under different energy conditions. Relying on energy harvesting to power WSNs presents a new challenge where nodes must aim to maximize their throughput within a short period of time when energy is available. Backoffs consume energy and retransmissions waste energy, both of which are not tolerable in WSNs powered by energy harvesting.

One of the first MAC protocols designed specifically for EH WSNs is a probabilistic polling scheme $[4,5]$ that aims to achieve fairness among different devices while maintaining acceptable throughput, and used realistic measurement data of solar energy harvesting in their simulations. The On Demand Medium Access Control (ODMAC) [6] protocol aims to maximize the network performance during the short period when there is sufficient energy on a node for sustainable operation. While the protocol has been extended to consider multi-hop scenarios [7], it has not addressed the issue of the strong correlation in energy harvesting rates among neighbouring nodes that can lead to serious contention. With severe energy constraints, it is desirable to maximize the usage of any available energy and Lei et al. [8] propose an information value threshold set based on energy level to let devices decide whether to transmit valuable packets or discard unimportant packets. Most, if not all, of the reported works are based on the harvesting solar energy but not vibration energy.

A key design consideration of WSNs using EH is the characteristics of the energy source. Ho et al. [9] provide two Markovian models to predict different harvesting energy sources. The first is used to predict stable energy sources such as solar energy, and the second is for unstable energy sources, like, vibration energy. Both these models need a long measurement period to collect data that can be used later to predict future amounts of harvested energy. In our scenario, the energy source is very unique in that the vibration lasts only for a short period, not much longer than that duration of the earthquake. Therefore, the amount of energy that can be harvested is also very limited and must be used in the most optimal manner. While current leakage is a serious problem in energy harvesting networks [10], it is unlikely to be a significant factor in our system as the duration of network operation is short. Nevertheless, we have accounted for it by using measured data from real testbed experiments [11], and this includes the effects of current leakage; current leakage is proportional to energy level. i.e., the higher the energy, the more severe the leakage will be.

\section{Energy Model}

In this section, we discuss the energy model which includes the energy income (from Energy Harvesting) and energy usage (Energy Consumption) as shown in Fig. 1. Capacitors are used to store the energy from the piezoelectric vibration energy harvester (PEH); in our study, we chose the PEH25W produced by MIDE [12] which provided the the flexibility we needed for tuning the resonant frequency and we will briefly discuss the measurement process and results below [11]. For the energy consumption, we calculate the power dissipation by monitoring the voltage and current of the wireless sensor node, in particular, the microprocessor [13] and radio transceiver chip [14], which are the key power consumers.

\section{A. Energy Income}

Building vibration frequencies that result from earthquakes fall in the $0.5 \sim 2 \mathrm{~Hz}$ range which is much lower than the resonant frequencies of most commercially available as well as non-commercial vibration energy harvesters [15]. Many of these devices are pre-tuned for specific frequencies, e.g. $60 \mathrm{~Hz}$, as they are designed for harvesting energy from machinery which vibrate at these higher frequency ranges. In order to determine the energy harvesting efficiency, we set up an experiment, as shown in Fig. 3. To measure the energy harvest 


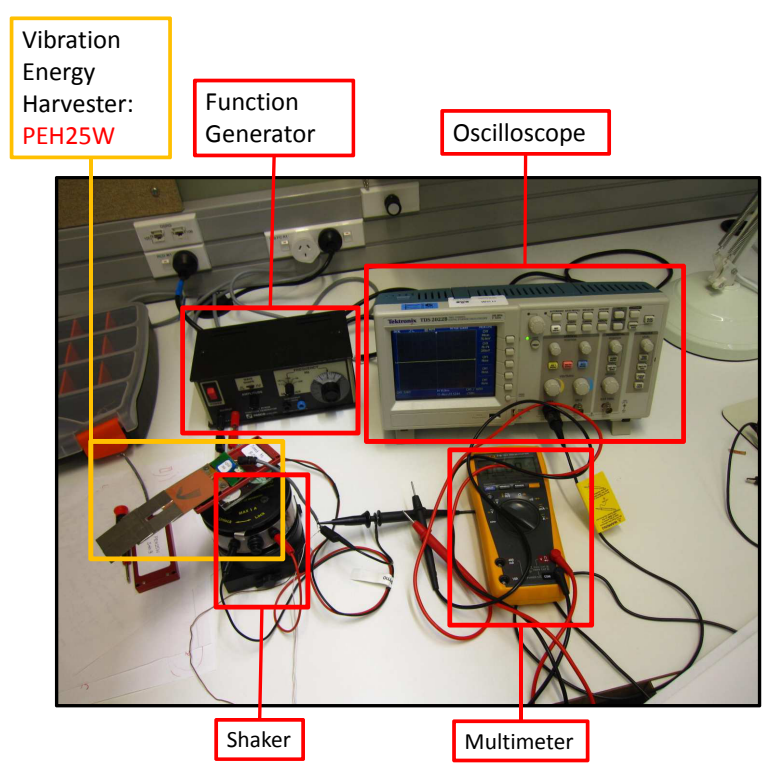

Fig. 3: Measurement Setting and Devices

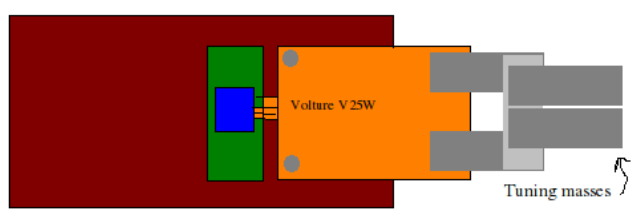

(a) $\mathrm{PEH} 25 \mathrm{~W}$ tuned for $8 \mathrm{~Hz}$

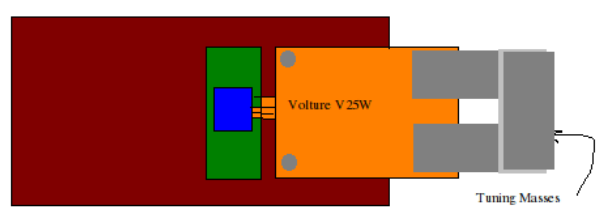

(b) $\mathrm{PEH} 25 \mathrm{~W}$ tuned for $12 \mathrm{~Hz}$

Fig. 4: PEH25W Tuning Masses and Beam Length

rate of the piezoelectric vibration energy harvester PEH25W, we use a function generator to drive the shaker which the PEH25W is mounted on. As the shaker moves, the beam oscillates and energy is generated which is stored in the capacitor on the PEH25W device. Only when the frequency of vibration approaches the resonant frequency will the energy harvesting rate reach the maximum value. Thus, we need to adjust the resonant frequency of the PEH $25 \mathrm{~W}$ by adding Tuning Masses and extending the length of the beam, as shown in Fig. 4 for resonant frequencies of $8 \mathrm{~Hz}$ and $12 \mathrm{~Hz}$; longer beam length for lower frequency.

We first measure the energy harvesting rates under fixed vibration frequency, around $7 \sim 8 \mathrm{~Hz}$ (minimum achievable resonant frequency without major modifications to the PEH25W), under different vibration amplitudes, i.e., different accelerations. We then observe that when the vibration acceleration

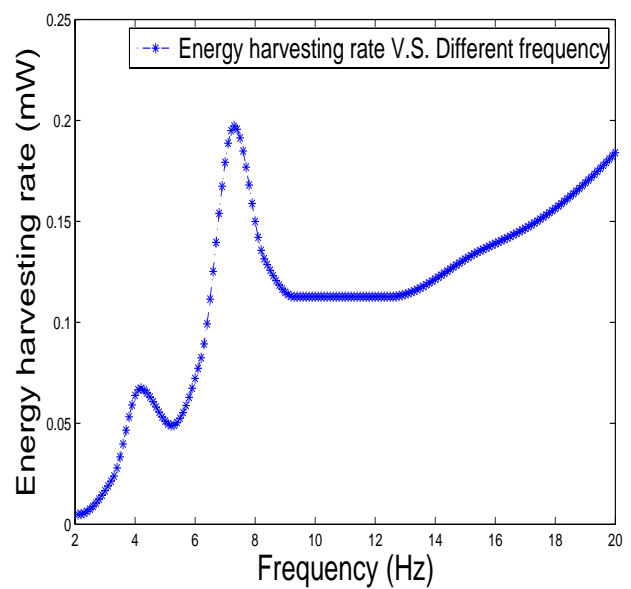

Fig. 5: Harvesting rate at resonant frequency of $8 \mathrm{~Hz}$

TABLE I: Energy Dissipation

\begin{tabular}{|l|l|l|l|l|}
\hline \multirow{2}{*}{ Symbol } & \multirow{2}{*}{ Meaning } & \multirow{2}{*}{ Measurement } & \multicolumn{2}{|c|}{ Datasheet Values } \\
\cline { 4 - 5 } & & & CC2520 & MSP430F2618 \\
\hline$P_{\text {init }}$ & Initialization & $162.936 \mathrm{~mW}$ & N/A & N/A \\
\hline$P_{T X}$ & Transmission & $135.036 \mathrm{~mW}$ & $120.96 \mathrm{~mW}$ & $1.08 \mathrm{~mW}$ \\
\hline$P_{R X}$ & Receiving & $78.912 \mathrm{~mW}$ & $66.6 \mathrm{~mW}$ & $1.08 \mathrm{~mW}$ \\
\hline$P_{L P}$ & Low power & $9.0252 \mathrm{~mW}$ & $0.000432 \mathrm{~mW}$ & $0.0032 \mathrm{~mW}$ \\
\hline$P_{T A}$ & Turnaround & $87.336 \mathrm{~mW}$ & N/A & N/A \\
\hline$E_{\text {min }}$ & $\begin{array}{l}\text { Minimum } \\
\text { operation }\end{array}$ & $1.6276 \mathrm{~mJ}$ & N/A & N/A \\
\hline
\end{tabular}

increases, the vibration energy harvesting rate predictably increases. For a resonant frequency fixed at $8 \mathrm{~Hz}$, using the configuration shown in Fig. 4a, the measured energy harvesting rate under different vibration frequency is shown in Fig. 5. In other respects, as the difference between the vibration frequency and the resonant frequency increases, the energy harvesting rate drops sharply. Thus, in order to maximize the harvested energy, we need to match the resonant frequency of vibration energy harvester to the earthquake vibration frequency. While improving the design of the VEH is not our goal, we need to ensure that we have a validated assumption, i.e. the ability to harvest sufficient energy from the vibrations of a building during an earthquake to power the wireless sensor node. In order to do this, we make further modifications to the PEH25W to lower the resonant frequency to the region of $0.5 \sim 2 \mathrm{~Hz}$, resulting the extended beam length that is evident in Fig. 3.

\section{B. Energy Usage}

Different operation modes of a device require different amounts of energy. To determine the energy usage, we focus on power dissipation in different operations of the wireless sensor node's components, primarily, key components that consume the most energy. These are shown in Table. I where CC2520 is the radio transceiver chip [13], and MSP430F2618 is the microprocessor [14].

The first operation is to power up each component in the 
device, that is, the initialization power; we denote it as $P_{\text {init }}$, and the average value is roughly $162.936 \mathrm{~mW} . P_{T X}$ and $P_{R X}$ are transmission power and receiving power, and the measured values are $135.036 \mathrm{~mW}$ and $78.912 \mathrm{~mW}$, respectively. We also list the values shown in the datasheets, and we find that measured results are slightly higher than those in the datasheets. The reason is that various other less-than-ideal effects like current leakage and power loss in energy transfer between components and power management unit are not accounted for in the datasheets. $P_{T A}$ is power to switch the transceiver from transmission mode to receiving mode or vice versa. When a device is idle, it will turn off its components to save its energy, going into low-power mode; we denote power dissipation in this mode as $P_{L P}$ and the measured value is $9.0252 \mathrm{~mW}$. This value is still higher than the value in the datasheets due to the same reason as above. These measurements corroborate with findings in other studies, e.g. [16] and [17].

\section{Analysis and Design Considerations}

We have confirmed that a VEH with resonant frequency tuned to the region of $0.5 \sim 2 \mathrm{~Hz}$ would be able to achieve maximum energy harvesting efficiency. However, in a real situation when an earthquake occurs, there will be multiple frequencies and amplitudes as shown in Fig. 6a [18, 19]. We can use the property of superposition to combine these different energy harvesting rates, and compute the corresponding amount of harvested energy. Combining the measured energy harvesting rates and historical earthquake data shown in Fig. 6a, we obtain Fig. 6b. In Fig. 6b, we note that harvested energy at different regions in an earthquake zone vary according to the distance of the EH device from the epicenter. Obviously, we find that harvested energy drops as the distance to epicenter increases; regions close to the epicenter have a higher chance of harvesting more energy than those further away. We use different colours to denote different amounts of harvested energy, corresponding to the number of transmission rounds achievable. More harvested energy means that devices can operate for longer durations (i.e., more transmission rounds) than other devices with lower harvested energy; the behaviour of devices in each transmission round will be described in Section IV-A. There remain various other factors can affect the harvested energy like building structure and the geography of the area [18]. Consequently, some regions are closer to the epicenter, yet the devices may not harvest enough energy to operate as there is lesser vibration. Harvested energy is accumulated in the capacitor(s) until device has acquired the minimum operating energy, then the stored energy is used to power up the device for operation - sense and transmit data.

Although the energy harvested from vibrations is significantly lower than the power consumption of devices, it is still sufficient for devices to operate, and transmit packets. This is shown later in section V-A. However, to ensure that the packets are delivered successfully, there are other obstacles to overcome, like the less-than-ideal channel conditions and the channel contention resulting from many nodes becoming

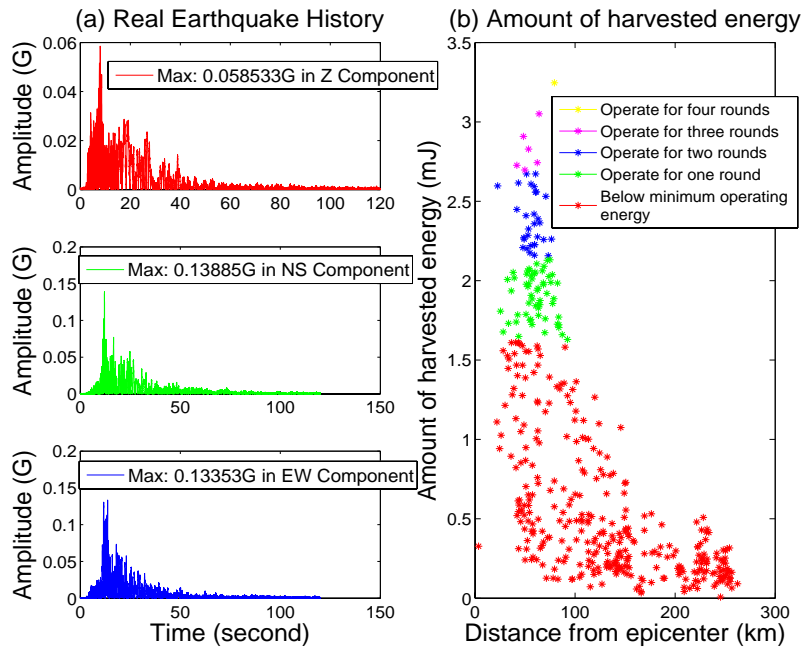

Fig. 6: Amount of harvested energy

operational almost simultaneously, causing packet collisions. In our SHM application, when an earthquake happens, all the devices charge up at this time, and try to transmit their packets leading to serious channel contention problems. When collisions happen, energy used in the transmission is wasted and, they cannot replenish energy until the next shockwave, which must come soon enough to replenish their energy before they run out due to leakage and other operating overheads. While the energy expended due to transfer loss and other operating overheads are inevitable, we can still minimize the effects. The duration of transmission and reception are fixed as long as the packet size is fixed, but power usage will be affected by selection of the backoff period. Unlike conventional MAC protocols where backoffs are used to reduce contention among nodes, a long backoff period during which energy is consumed can result in a node not having enough energy to transmit when the backoff period expires.

Thus, the IEEE 802.15.4 protocol that is designed for lowpower wireless networks has been found to be unsuitable for use in such applications. In section IV, we will briefly explain the operation of the IEEE802.15.4 protocol in our SHM application to validate this observation, and how we modify it to achieve better performance.

\section{SySTEM MODEL}

In this section, we model our problem and state the assumptions made in our analysis. As previously mentioned, a key feature of the SHM application is that devices within close proximity are likely to have similar information to report. Thus, in order to avoid transmitting similar information, we will divide the monitoring area into clusters. In each cluster, we only need to transmit one packet to report the status. Thus, once the packet has been successfully received by the AP (cf: Fig. 2), the AP will broadcast an acknowledgement (ACK) to all devices in the network. The devices within the same clusters will know that they do not need to transmit again because the status of this cluster has been reported. The 


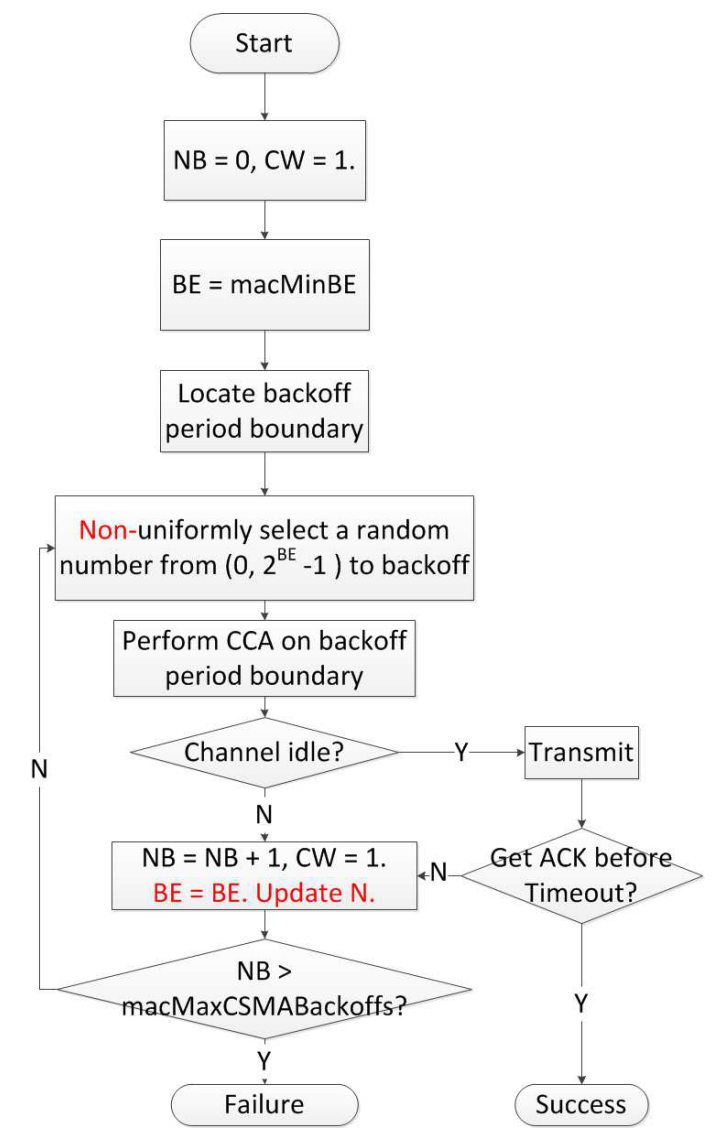

Fig. 7: Modified IEEE802.15.4 Protocol for EDEH WSN

number of clusters and the size of each cluster are system parameters which will be discussed later. We first describe the device functionality and the modification that we make to the IEEE 802.15.4 protocol, shown in red in Fig. 7.

\section{A. Device Functionality}

In the event of an earthquake, all devices begin harvesting and accumulating energy in their capacitors. Once sufficient energy is obtained, devices will turn on their microprocessors and transceivers to perform synchronization and other necessary operations; the devices will continue to harvest energy until the vibrations are over. Once synchronization is completed, devices backoff several time slots to avoid collision with other devices. During the backoff period, a device will stay in low power mode to save energy. After the backoff period expires, the device activates the transceiver and performs the Clear Channel Assessment (CCA) procedure to check whether channel is idle or not, and increments the number of backoff trials (NB) by one. If the channel is idle and Contention Window (CW) is zero, it will transmit packets and wait for the ACK from the AP. If ACK is not received or the channel is busy, the device will turn off its radio to save power and wait until the start of the next transmission round. Devices will repeat the procedure until they run out their energy or receive an ACK.

\section{B. Protocol Description}

According to the original IEEE 802.15.4 protocol, once a device fails at a transmission attempt, often due to collision, the device will increase its backoff exponent (BE). Thus, the device has more time slots to backoff, thereby decreasing the chance of collision. However, due to the design considerations described in Section III-C, extending the backoff timer may not be a good choice to solve this contention problem; furthermore, waiting will also deplete the limited energy supply. Thus, the modification is not to increase the BE, i.e. retain its value as shown in Fig. 7. In the original IEEE 802.15.4 protocol, each device randomly selects a backoff timer within the range of BE uniformly (i.e., each time slot has the same probability to be selected). Instead, we assign different time slots with different probabilities of being selected, i.e. nonuniform probability. The main idea behind solving contention problems in IEEE 802.15.4 protocol is to use a larger BE and spread access of devices over a longer time, this reduces the chance of collision at the cost of increased delay. Furthermore, this method may take several cycles to find a better BE value, and in a network relying on energy harvesting for power, devices do not have so much energy to try and find this $\mathrm{BE}$ value. In our modified protocol, we aim to make an optimal backoff time slot selection based on current contention condition, namely, how many devices are contending the channel, given that the probability to select each time slot, may vary as contending condition changes.

Here, we refer to our proposed algorithm as the optimal backoff time slot selection algorithm. Suppose there are N devices which are contending to transmit packets and $\mathrm{T}$ backoff timers that could be selected, with probabilities $p_{1}, p_{2}$, $\ldots, p_{T-1}, p_{T}$ of selecting each backoff timer. For instance, $p_{1}$ represents the probability of picking the first available time slot to transmit. Thus, our objective function $S_{p}(N, T)$ is modeled as follows:

$$
\begin{aligned}
S_{p}(N, T)= & N p_{1}\left(1-p_{1}\right)^{N-1}+N p_{2}\left(1-p_{1}-p_{2}\right)^{N-1} \\
& +\cdots+N p_{T-1}\left(1-p_{1}-\cdots-p_{T-1}\right)^{N-1} \\
= & N \sum_{i=1}^{T-1} p_{i}\left(1-\sum_{j=1}^{i} p_{j}\right)
\end{aligned}
$$

Thus,

$$
\frac{S_{p}(N, T)}{N}=\sum_{i=1}^{T-1} p_{i}\left(1-\sum_{j=1}^{i} p_{j}\right)^{N-1}
$$

$S_{p}(N, T)$ represents the case of one node selecting a time slot to transmit, and no any other node colliding with it; in other words, it is the probability of a successful transmission. To obtain the maximum probability of successful transmission, we find the first derivative of $S_{p}(N, T)$ with respect to each $p_{i}$ and equate it to 0 , as follows:

$$
\frac{\partial}{\partial p_{i}}\left(\frac{S_{p}(N, T)}{N}\right)=0
$$




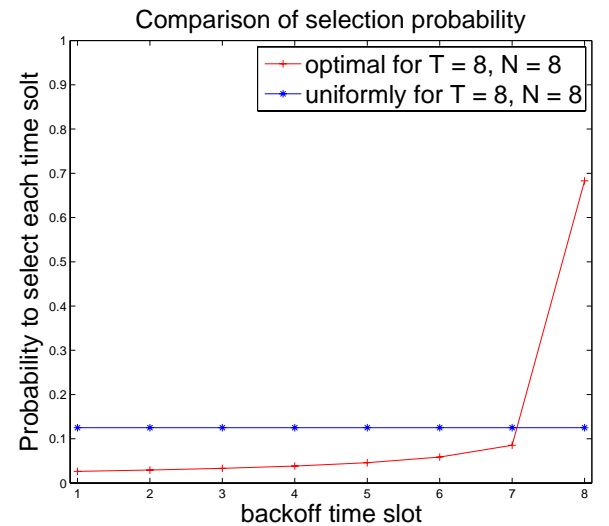

Fig. 8: Probability to select each backoff time slot

By mathematical induction on $i$, we obtain:

$$
(N-f(N, i)) p_{T-i}=(1-f(N, i))\left(1-\sum_{k=1}^{T-(i+1)} p_{k}\right)
$$

The derivation process from Eqn. (1) to (3) has been shown by Y. C. Tay et al [20], and the probability which could maximize the success probability of each time slot is given by:

$$
p_{T-i}=\frac{1-f(N, i)}{N-f(N, i)}\left(1-\sum_{k=1}^{T-(i+1)} p_{k}\right)
$$

where $p_{T-i}$ is the probability of selecting the time slot $T-i$, $f(N, i)$ is maximum conditional success probability when no node chooses any slot before $T-i+1$, and $N$ is the number of contending devices. As Fig. 7 shows, $N$ is updated in each transmission round. An example scenario is shown in Fig. 8. A device will first generate a random number between 0 and 1 , and compare the number it generated with the cumulative distribution function (CDF) of the probabilities $p_{1}, p_{2}, \ldots$, $p_{T-1}, p_{T}$. If the generated number is smaller than the location it falls in the CDF area, the device will select the upper bound value of this area to be the backoff timer. Suppose BE is 3 , the number of contending devices is 16 and a device generates a random number 0.13 . For the IEEE 802.15.4 protocol, where $p_{1}=p_{2}=\ldots p_{8}=0.125$, the value of 0.13 falls between 0.125 and 0.25 which are $p_{1}$ and $\left(p_{1}+p_{2}\right)$ respectively. Thus, the device will select the second available time slot to transmit. When the device is ready to transmit, it will perform CCA, and only if the channel is idle will it turn the radio to transmit (TX) mode and transmit. Following the transmission, if no ACK is received (or the device gets a NACK), it means the packet was not successfully received by the AP, and the device will start the next transmission round, repeating the same sequence of actions. Each action will incur the energy cost listed in Table I.

\section{Clustering}

In the previous section, we have shown that the optimal backoff time slot selection in each transmission round depends on the number of contending devices. We now describe the clustering approach and its effect on the system performance by reducing the number of contending nodes in each round. We note that the number of clusters in the network is determined by the proximity of devices to one another or building structure; the decision on which devices to form a cluster may be determined by domain experts, e.g. structural engineers. In each transmission round, at most one cluster can successfully transmit its packet. Other clusters should wait for the next transmission round. Thus, the successful probability of each cluster is given by Eqn. (5), where $S_{p_{x}}(N, M, T)$ is the success probability of an arbitrary cluster (cluster $x$ ), $N$ is the number of contending devices, $M$ is the number of clusters that still need to transmit packets, and $T$ is the number of time slots that can be selected as backoff duration. $f_{p_{x}}(N, M, T-1)$ is maximum conditional success probability when no device chooses any slot before time slot $2 . N_{x}$ is the number of devices in cluster $x, N_{s}$ is the number of devices in the successful cluster of the current round, and $N_{\text {out }}$ is the number of devices in outage.

The first term of the R.H.S. of Eqn. (5) represents the condition when the channel is idle and the available time slots will decrease by one because no device accessed this channel. The second and third terms represent the conditions when only one device successfully accessed the channel, the difference being that the second term refers to the case where the successful device belongs to the same cluster. Thus, other devices in the cluster do not need to transmit. On the other hand, if the successful transmission is in another cluster, meaning that the remaining clusters still need to contend for the next round. The last term represents a collision condition, and thus all the devices with enough remaining energy will compete in the next round, using the same procedure. After the completion of each transmission round, all the devices will be updated by the access point via an ACK or NACK to know how many devices are still contending for the channel.

\section{Performance Evaluation}

In this section, we verify the analytical results of Section IV by using simulation. We first obtain some empirical data using the procedure described in Section III as our input parameter to our simulation, and show those analytical results corroborates with the simulation results.

\section{A. Simulation parameters and metrics}

Table II lists the network parameters used in the simulation. The period of unit backoff is $0.32 \mathrm{~ms} . S_{D A T A}$ and $S_{A C K}$ are the size of data and ACK packets, respectively, with values 51 bytes and 11 bytes. $T_{i n i t}$ is the node initialization time. $T_{C C A}$ and $T_{T A}$ are the times required by the radio transceiver to perform CCA and switch from TX to RX mode or vice versa. $T_{D A T A}$ and $T_{A C K}$ are the times that a device spends on transmitting and receiving ACKs. macMaxCSMABackoffs and macMaxBE are the maximum value of $\mathrm{NB}$ and $\mathrm{BE}$, respectively. The parameters in Table II follow the settings in [21]. The theoretical energy consumption is given by:

$$
E_{c o n}=\mathbf{P} \mathbf{T}
$$




$$
\begin{aligned}
& S_{p_{x}}(N, M, T)=\left(1-p_{1}\right) f_{p_{x}}(N, M, T-1)+N_{x} p_{1}\left(1-p_{1}\right)^{N-1}+ \\
& \quad\left(N-N_{x}\right) p_{1}\left(1-p_{1}\right)^{N-1} S_{p_{x}}\left(\left(N-\left(N_{s} \cup N_{\text {out }}\right)\right), M-1, T\right)+\sum_{i=2}^{N}\left(\begin{array}{c}
N \\
i
\end{array}\right) p_{1}{ }^{i}\left(1-p_{1}\right)^{N-i} S_{p_{x}}\left(\left(N-\left(N_{\text {out }}\right)\right), M, T\right)
\end{aligned}
$$

\begin{tabular}{|c|c|c|}
\hline \multicolumn{2}{|c|}{ Parameter } & Setting \\
\hline \multicolumn{2}{|c|}{ aUnitBackoffPeriod } & $0.32 \mathrm{~ms}$ \\
\hline \multicolumn{2}{|c|}{$S_{D A T A}$} & 51 bytes \\
\hline \multicolumn{2}{|c|}{$S_{A C K}$} & 11 bytes \\
\hline \multicolumn{2}{|c|}{$T_{\text {init }}$} & $1.4 \mathrm{~ms}$ \\
\hline \multicolumn{2}{|c|}{$T_{C C A}$} & $0.128 \mathrm{~ms}$ \\
\hline \multicolumn{2}{|c|}{$T_{T A}$} & $0.192 \mathrm{~ms}$ \\
\hline \multicolumn{2}{|c|}{$T_{D A T A}$} & $1.344 \mathrm{~ms}$ \\
\hline \multicolumn{2}{|c|}{$T_{A C K}$} & $0.352 \mathrm{~ms}$ \\
\hline \multicolumn{2}{|c|}{ macMaxBE } & 5 \\
\hline \multicolumn{2}{|c|}{ macMaxCSMABackoffs } & 4 \\
\hline \multicolumn{3}{|c|}{ Cluster information } \\
\hline Cluster \# & Number of 1 & Harvested Energy \\
\hline 1 & 128 & $2.1 \mathrm{~mJ}$ \\
\hline 2 & 256 & $2.1 \mathrm{~mJ}$ \\
\hline 3 & 128 & $2.6 \mathrm{~mJ}$ \\
\hline
\end{tabular}

TABLE II: Network Parameter settings

where $E_{c o n}$ denotes the total energy consumption. Power vector $\mathbf{P}$ consists of power consumption entries to complete one transmission, while time vector $\mathbf{T}$ consists of the corresponding time entries. If we assess the energy depletion of one successful transmission without backoff, then

$$
\begin{aligned}
\mathbf{P} & =\left[\begin{array}{lllllll}
P_{\text {init }} & P_{R X} & P_{T A} & P_{T X} & P_{T A} & P_{R X} & P_{L P}
\end{array}\right], \text { and } \\
\mathbf{T}^{T} & =\left[\begin{array}{lllllll}
T_{\text {init }} & T_{C C A} & T_{T A} & T_{D A T A} & T_{T A} & T_{A C K} & T_{L P}
\end{array}\right] .
\end{aligned}
$$

The equation results in $0.48 \mathrm{~mJ}$ energy consumption, when the parameter values from Table I and II are substituted into these two vectors. $T_{L P}$, which denotes the time spent in low-power mode, could be ignored and set to zero, since we just assessed the energy cost for one successful transmission without lowpower mode. Moreover, Table I and Fig. 6(b) show that the minimum operation energy within the device is $1.6276 \mathrm{~mJ}$ at least. In the case of the harvested energy up to $1.6276 \mathrm{~mJ}$, the energy is able to afford the transmission energy $0.48 \mathrm{~mJ}$. This theoretically supports evidence that the harvested energy within the active device is enough to perform at least one transmission, when the amount of harvested energy is more then minimum operation level. Base on the data shown in the Fig. 6(b), the amount of harvested energy may be up to $3 \mathrm{~mJ}$ or more, when the devices are located $80 \mathrm{~km}$ away from the epicenter. These devices hold more energy to do more transmission rounds. Naturally more transmission rounds provide more successful opportunities for AP to collect the earthquake data.

Regarding clustering, we show the case of 3 clusters and 512 devices in the network. In each cluster, the number of devices and mean harvested energy per device are listed in Table II. Using these parameters, we show the variation of success probability for each cluster, which is defined as the expected value of transmitted packets that can be successfully received by the AP for each cluster.

\section{B. Discussions}

We will examine the effect of different number of devices in each cluster and different levels of harvested energy in each cluster. As listed in Table II, cluster \#1 contains 128 devices that harvest $2.1 \mathrm{~mJ}$ each; cluster \#2 contains 256 devices and the devices also harvest $2.1 \mathrm{~mJ}$ each. Cluster \#3 contains 128 devices and each device harvests $2.6 \mathrm{~mJ}$, more than those in the previous two clusters. In practice, it is hard to rely on only one device to harvest enough energy and sense in an earthquake event. That is why more devices are deployed for redundancy and robustness. Even some devices are nonoperational, we do not need to replace them, as long as other devices are still operating, and protocol can function. It is particularly noteworthy that the advantage of proposed protocol will diminish significantly, if the number of device is few, e.g. numbering two or three. Under such conditions, channel contention is negligible. The originally IEEE 802.15.4 protocol could be seen as a special case in the proposed protocol when number of devices is two.

In Fig. 9, the success probability of cluster \#1 is the lowest among the three clusters. The reason is that there are fewer devices and less harvested energy overall, and as such it has no advantage in contending the channel. Consequently, its success probability is lower than those of the other two clusters. For cluster \#2, it has twice the number of devices, and we observe that the success probability is roughly twice of that of cluster \#1. However, the success probability of cluster \#2 is still lower than cluster \#3, which has the highest level of harvested energy. Although cluster \#3 has fewer devices than cluster \#2, it still has more energy than cluster \#2. All devices within cluster \#3 have a higher chance of transmission than those in clusters \#2 and \#1 which is why the success probability of cluster \#3 is the highest among the three. Intuitively, that is the desired outcome as cluster \#3 nodes harvested more energy because their part of the building experienced greater vibrations, has a higher risk of damage and hence their data are more critical. The original IEEE 802.15.4 protocol, on the other hand, could not support a huge number of devices simultaneously accessing the channel, resulting in success probabilities that are close to zero for all cases.

\section{CONCLUSION}

This paper addresses a special case of SHM applications that focuses on the rare event occurrence, namely, earthquakes, and combines with the unique properties of EH technologies. When an earthquake occurs, all sensing devices which are 


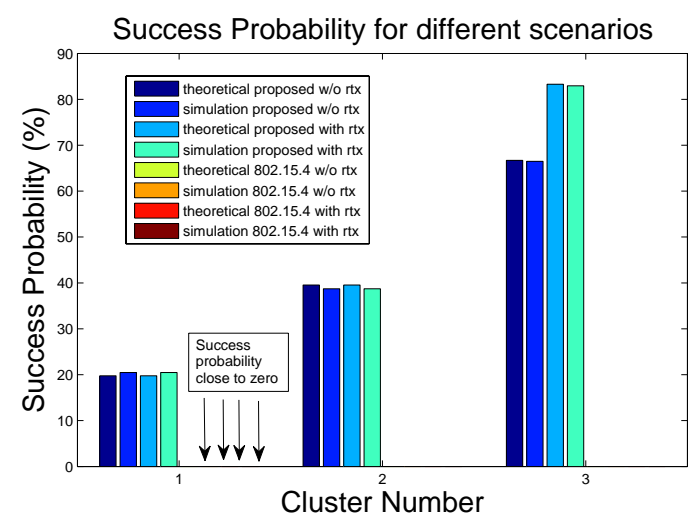

Fig. 9: Success probability for different number of clusters

powered by vibration energy harvesting become active simultaneously leading to severe channel contention problems. This is coupled with the scarce amounts of energy that can be harvested from the short event occurrence time. Based on these observations, we propose the EDEH MAC protocol to address channel contention under stringent energy constraints. Our performance analysis corroborates with simulation results using real measurement data as input. Moving forward, there are still outstanding issues which warrant further study. E.g., devices with higher amounts of harvested energy, after completion of their tasks, can use their remaining energy to help other devices transmit their data, similar to cooperative networking.

\section{REFERENCES}

[1] W.K.G. Seah, Z.A. Eu, and H.P. Tan. Wireless sensor networks powered by ambient energy harvesting (WSNHEAP) - Survey and Challenges. In Proc of the 1st Int'l Conf on Wireless Communication, Vehicular Technology, Information Theory and Aerospace \& Electronic Systems Technology (Wireless VITAE), Aalborg, Denmark, 2009.

[2] A. Kansal and M.B. Srivastava. An environmental energy harvesting framework for sensor networks. In Proc of the Int'l Symp on Low Power Electronics and Design., pages 481-486, 2003.

[3] A. Kansal, J. Hsu, S. Zahedi, and M.B. Srivastava. Power management in energy harvesting sensor networks. Transactions on Embedded Computing Systems (TECS), 6(4):32, 2007.

[4] Z.A. Eu, W.K.G. Seah, and H.P. Tan. A study of MAC schemes for wireless sensor networks powered by ambient energy harvesting. In Proc of the 4th Annual Int'l Conf on Wireless Internet (WICON), Maui, HI, USA, 17-19 Nov. 2008.

[5] Z.A. Eu, H.P. Tan, and W.K.G. Seah. Design and performance analysis of MAC schemes for Wireless Sensor Networks Powered by Ambient Energy Harvesting. Ad Hoc Networks, 9(3):300-323, 2011.

[6] X. Fafoutis and N. Dragoni. ODMAC: an on-demand MAC protocol for energy harvesting - wireless sensor networks. In Proc of the 8th ACM Symposium on
Performance Evaluation of Wireless Ad hoc, Sensor, and Ubiquitous Networks (PE-WASUN), pages 49-56, Miami, Florida, USA, 31 Oct - 4 Nov 2011.

[7] X. Fafoutis and N. Dragoni. Adaptive media access control for energy harvesting - wireless sensor networks. In Proc of the 9th Int'l Conf on Networked Sensing Systems (INSS), Antwerp, Belgium, 11-14 June 2012.

[8] J. Lei, R. Yates, and L. Greenstein. A generic model for optimizing single-hop transmission policy of replenishable sensors. IEEE Transactions on Wireless Communications, 8(2):547-551, 2009.

[9] C.K. Ho, P.D. Khoa, and P.C. Ming. Markovian models for harvested energy in wireless communications. In Proc of the IEEE Int'l Conf on Communication Systems (ICCS), Singapore, 17-19 November 2010.

[10] T. Zhu, Z. Zhong, Y. Gu, T. He, and Z.L. Zhang. Leakage-aware energy synchronization for wireless sensor networks. In Proc of the 7th Int'l Conf on Mobile systems, applications, and services (MobiSys), pages 319-332, Kraków, Poland, 2009. ACM.

[11] Y.H. Tham. Summer Internship Report: Design and Experimentation of a Wireless Sensor Network Node Powered by Vibration Energy. Technical Report ECSTR1215, Victoria University of Wellington, May 2012.

[12] Mide Volture Systems. PEH25W Vibration Energy Harvesters, 2010.

[13] Texas Instruments Inc. MSP430F261x Mixed Signal Microcontroller Datasheet, December 2011.

[14] Texas Instruments Inc. CC2520 2.4GHz IEEE802.15.4 / Zigbee $\mathbb{R}$ RF Transceiver Datasheet, December 2007.

[15] S.P. Beeby, M.J. Tudor, and N.M. White. Energy harvesting vibration sources for microsystems applications. Measurement Science and Technology, 17(12):R175R195, December 2006.

[16] P. Yu, L. Qinghua, and P. Xiyuan. The design of lowpower wireless sensor node. In Proc of the IEEE Instrumentation and Measurement Technology Conf $\left(I^{2} M T C\right)$, Austin, TX, USA, 3-6 May 2010.

[17] J.P. Olds and W.K.G. Seah. Design of an active radio frequency powered multi-hop wireless sensor network. In Proc of the 7th IEEE Conf on Industrial Electronics and Applications (ICIEA), Singapore, 18-20 July 2012.

[18] C.C. Chiang. Seismic behaviour of building with midstory isolation. Master's thesis, National Taiwan University, June 2007.

[19] TW Central Weather Bureau. Earthquake damage reports. http://www.cwb.gov.tw/V7/earthquake/damage_eq.htm, last accessed: 22 April 2012.

[20] Y. C. Tay, K. Jamieson, and H. Balakrishnan. Collisionminimizing csma and its applications to wireless sensor networks. IEEE Journal on Selected Areas in Communications, 22(6):1048-1057, Aug. 2004.

[21] IEEE802.15.4 Stds., Wireless Medium Access Control (MAC) and Physical Layer (PHY) Specifications for LowRate Wireless Personal Area Networks (WPAN), 2006. 